\title{
Hydroxychloroquine and Coronavirus Disease 2019: A Systematic Review of a Scientific Failure
}

\author{
Stav Rakedzon, M.D. ${ }^{*}$, Yara Khoury, M.D. ${ }^{1}$, Gilad Rozenberg, M.D. ${ }^{1}$, and Ami \\ Neuberger, M.D. ${ }^{1,2,3}$ \\ ${ }^{\prime}$ Department of Internal Medicine, Rambam Health Care Campus, Haifa, Israel; ${ }^{2}$ Division of Infectious \\ Diseases, Rambam Health Care Campus, Haifa, Israel; and ${ }^{3}$ The Ruth $\mathcal{E}^{2}$ Bruce Rappaport Faculty of \\ Medicine, Technion-Israel Institute of Technology, Haifa, Israel
}

\begin{abstract}
Introduction: Hydroxychloroquine (HCQ) emerged early in the course of the coronavirus disease 2019 (COVID-19) pandemic as a possible drug with potential therapeutic and prophylactic benefits. It was quickly adopted in China, Europe, and the USA. We systematically reviewed the existing clinical evidence of HCQ use for the prevention and treatment of COVID-19.
\end{abstract}

Methods: We screened for clinical studies describing HCQ administration to treat or prevent COVID-19 in PubMed. We included randomized controlled trials (RCTs), non-randomized comparative cohorts, and case series studies that had all undergone peer review.

Results: A total of 623 studies were screened; 17 studies evaluating HCQ treatment were included. A total of 13 were observational studies, and 4 were RCTs. In terms of effect on mortality rates, observational studies provided conflicting results. As a whole, RCTs, including one large British RCT that has not yet been

\footnotetext{
Abbreviations: AZ, azithromycin; COVID-19, coronavirus disease 2019; EUA, emergency use authorization; FDA, US Food and Drug Administration; HCQ, hydroxychloroquine; ICU, intensive care unit; ISO, International Organization for Standardization; RCT, randomized controlled trial; SARS-CoV-2, severe acute respiratory syndrome coronavirus-2.

Citation: Rakedzon S, Khoury Y, Rozenberg G, Neuberger A. Hydroxychloroquine and Coronavirus Disease 2019: A Systematic Review of a Scientific Failure. Rambam Maimonides Med J 2020;11 (3):eo025. Review. doi:10.5041/RMMJ.10416

Copyright: (C) 2020 Rakedzon et al. This is an open-access article. All its content, except where otherwise noted, is distributed under the terms of the Creative Commons Attribution License (http://creativecommons.org/licenses/by/3.0), which permits unrestricted use, distribution, and reproduction in any medium, provided the original work is properly cited.

Conflict of interest: No potential conflict of interest relevant to this article was reported.

* To whom correspondence should be addressed. E-mail: stav.rakedzon@gmail.com
} 
published, showed no significant effect of HCQ on mortality rates, clinical cure, and virologic response. The use of HCQ as a post-exposure prophylactic agent was found to be ineffective in one RCT.

Conclusion: There is no evidence supporting HCQ for prophylaxis or treatment of COVID-19. Many observational trials were methodologically flawed. Scientific efforts have been disappointingly fragmented, and well-conducted trials have only recently been completed, more than 7 months and 600,000 deaths into the pandemic.

KEY WORDS: Chloroquine, coronavirus, COVID-19, hydroxychloroquine, pandemic, review

\section{INTRODUCTION}

The coronavirus disease 2019 (COVID-19) pandemic is a global healthcare crisis that has already cost more than 600,000 lives worldwide, and is associated with enormous economic, geopolitical, and social burdens. ${ }^{1}$

As no drug was known to be effective at the onset of the pandemic, efforts were aimed at repurposing existing agents to treat severe acute respiratory syndrome coronavirus-2 (SARS-CoV-2). Very early on in the course of the pandemic, chloroquine and its less toxic derivative hydroxychloroquine (HCQ) emerged as possible candidates and were quickly adopted in China, where COVID-19 had initially emerged.

Chloroquine and HCQ have traditionally been used to treat chronic inflammatory diseases, malaria, and Q fever. In vitro SARS-CoV-2 inhibition was thought to be caused by interference with viral entry to host cells and the reduction of viral replication. ${ }^{2-5}$ Additionally, established immunomodulatory effects of HCQ were considered to be potentially beneficial in treating patients suffering "cytokine storm" due to COVID-19. As the pandemic progressed, the higher potency of HCQ against SARS-CoV-2 (as compared to chloroquine), ${ }^{6,7}$ together with its improved safety profile, made HCQ part of national guidelines in many countries. A heated medical and political debate regarding the theoretical benefits and possible adverse events of HCQ use for COVID-19 has been going on for months. Studies have been retracted from major medical journals, and authors were blamed for methodological inconsistencies and lack of transparency. Even now, 7 months into the global pandemic, conclusive studies have not yet been published.

This paper therefore provides a systematic review of the existing clinical evidence regarding the use of HCQ for the prevention and treatment of
COVID-19, and we discuss some of the factors that led to this failure of the scientific community.

\section{METHODS}

\section{Type of Studies}

For the purpose of review, we looked for clinical studies that administered HCQ to treat or prevent COVID-19. Inclusion criteria were: patients of all ages who had been diagnosed with COVID-19 and were treated with hydroxychloroquine, and were participants in randomized controlled trials (RCTs), non-randomized comparative cohort studies, and/or case series studies. Any study not meeting the above criteria, or that included patients who did not have COVID-19, or were taking hydroxychloroquine for other indications, and/or infections other than COVID-19, were excluded.

\section{Types of Outcome Measures}

Clinically relevant outcomes such as mortality rates, rates of admission, length of hospital stay, need for mechanical ventilation, and disease severity were noted. In addition, we included virologic parameters such as viral clearance times, viral shedding duration, and viral load.

\section{Search Methods for Identification of Studies}

We conducted a systematic electronic literature search within PubMed. We used one of the following search terms: "COVID-19" and/or "coronavirus," combined with the drug name "hydroxychloroquine." We also used the references of retrieved papers, including reviews and systematic reviews, to identify further studies. Two reviewers (authors S.R. and G.R.) independently screened all studies published before 20 June 2020 . We excluded all initially identified and retrieved articles that did not fulfill the inclusion criteria. In case of disagreement, a third reviewer acted as arbitrator (author A.N.). 
Reporting was performed in accordance with the Preferred Reporting Items for Systematic Review and Meta-Analyses (PRISMA).

\section{RESULTS}

\section{Studies Evaluating HCQ for the Treatment of COVID-19}

Our systematic search yielded 623 studies from PubMed. Overall, we included 13 observational studies describing outcomes of 8,967 patients treated with HCQ (Table 1) ${ }^{8-20}$ and 4 RCTs (one of which was a preliminary report of an unpublished study ${ }^{22}$ ) that described the outcomes of 2,299 patients treated with HCQ (Table 2). ${ }^{23-25,37}$ We describe separately several studies (apart from the 17 studies that were formally included in the analysis) with severe methodological issues (Table 3). ${ }^{26-29}$

\section{Observational studies}

Two studies were uncontrolled, non-comparative, observational studies from France that included patients with laboratory-confirmed COVID-19 who were treated with HCQ and azithromycin (AZ), without a control group. ${ }^{8,9}$ In the trial of Gautret et al. clinical improvement was noted in $97.5 \%$ of patients $(n=80)$; all patients were quickly discharged, and a rapid decline in nasopharyngeal viral load was reported. ${ }^{8}$ The second study, by Million et al., noted prolonged viral carriage in $4.4 \%$ of patients $(n=$ 1,061 ) and at least one adverse outcome (either death or transfer to an intensive care unit, or hospitalization for at least 10 days) in $4.3 \% .9$

In addition to the lack of a control group, there were other major methodological flaws in these two studies. Patients were relatively young, with median ages of 52.5 and 43 years in the first ${ }^{8}$ and second 9 studies, respectively. Such young patients have a very low risk for COVID-19 complications, even without any treatment, and post-treatment followup time was inadequate (in some patients, only 6 days).

Nine additional observational studies compared HCQ, with or without AZ, to control groups that did not receive HCQ. ${ }^{10-18}$ Four of these studies showed no significant mortality rate differences for patients receiving HCQ compared to controls. ${ }^{10-12,17}$ In one study, the risk for all-cause mortality was significantly higher among HCQ-treated patients compared to patients not treated with HCQ (adjusted hazard ratio $1.83 ; 95 \%$ CI $1.16-2.89 ; P=0.009$ ); however, mortality was similar when patients treated with $\mathrm{HCQ}+\mathrm{AZ}$ were compared to control patients (adjusted hazard ratio 1.13; 95\% CI $0.8-2.15$; $P=0.28) .{ }^{13}$ In three studies, two of which were relatively large, a significant reduction in mortality rates was observed in the HCQ group compared to the control group. ${ }^{14,15,18}$ Several inherent methodological flaws are worth mentioning in these trials (Table 1). In one study, for example, the mean age of patients in the treatment group was 5 years higher than in the control group, and there was a major difference between the number of patients receiving steroid treatment (a treatment that has been shown to be beneficial for some patients) in the HCQ group as compared with the non-HCQ groups $(74.3 \%$ in $\mathrm{HCQ}+\mathrm{AZ}, 78.9 \%$ in HCQ alone, $38.8 \%$ in AZ alone, and $35.7 \%$ in the control group). ${ }^{15}$

Two observational studies compared HCQ to other drugs.19,20 Kim et al. compared HCQ to lopinavir-ritonavir as a COVID-19 treatment; these authors observed a faster conversion of viral RNA in polymerase chain reaction (PCR)-based assays among patients treated with lopinavir-ritonavir. ${ }^{19}$ Vahedi et al. assessed HCQ as part of a broader treatment regimen containing multiple drugs; no beneficial change in oxygen saturation, body temperature, and C-reactive protein levels were observed. ${ }^{20}$

\section{Observational studies with severe methodological issues}

Gautret and colleagues conducted an open-label non-randomized trial in France; they found significantly higher numbers of virologically cured patients at 6 days in the group of patients treated with HCQ compared to the control group (70\% versus $12.5 \%$, respectively, $P=0.001) .{ }^{26}$ However, this study had several severe methodological flaws. Firstly, there was a major difference in group size and mean patient ages (26 patients, with mean age of 37.3 years, in the treatment group versus 16 patients, with mean age of 51.2 years, in the control group). Secondly, 6 patients were strangely excluded from the HCQ treatment groups, for example, due to a death that was erroneously defined as "lost to follow-up," $(n=1)$, transfer to an intensive care unit $(n=3)$, adverse effects of treatment $(n=1)$, and early recovery $(n=1)$. Finally, the control group $(n=16)$ had no follow-up data for 5 patients on day 6 , nor on day 5 for 2 of the same patients, resulting in an exaggeration of estimated COVID-19 positivity. 
Table 1. Summary of Observational Studies Evaluating HCQ for COVID-19 Treatment.

\begin{tabular}{|c|c|c|c|c|}
\hline Study Design & Pts & Study Groups & $\begin{array}{c}\text { Results: Primary/Secondary* } \\
\text { Outcomes }\end{array}$ & Methodological Issues \\
\hline $\begin{array}{l}\text { Comparative } \\
\text { observational } \\
\text { study }^{18}\end{array}$ & 3737 & $\begin{array}{l}\text { 1. } \mathrm{HCQ}+\mathrm{AZ} \\
\text { 2. Others sub- } \\
\text { divided to: } \\
\text { A. HCQ-AZ <3 days } \\
\text { B. HCQ } \\
\text { C. AZ } \\
\text { D. SOC }\end{array}$ & $\begin{array}{l}\text { HCQ+AZ treatment compared to } \\
\text { control: } \\
\text { - } \downarrow \text { Risk of death or transfer to } \\
\text { ICU (HR 0.18; } 95 \% \mathrm{Cl} 0.11 \text { - } \\
0.27)^{*} \\
\text { - } \downarrow \text { Hospitalization } \geq 10 \text { days (OR } \\
0.38 ; 95 \% \mathrm{Cl} 0.27-0.54 \text { ) } \\
\text { - Shorter viral shedding duration } \\
\text { in HCQ+AZ compared with all } \\
\text { other subdivided groups, } \\
\text { compared with SOC (HR } 1.29 \text {; } \\
\text { 95\% Cl 1.17-1.42) }\end{array}$ & $\begin{array}{l}\text { - Unequal group sizes (Tx } \\
\text { Pts } n=3,119 \text { vs “Other" } \\
\text { Pts } n=618 ; \text { with many } \\
\text { Txs and no pre-specified } \\
\text { protocol }\end{array}$ \\
\hline $\begin{array}{l}\text { Comparative } \\
\text { observational } \\
\text { study }^{15}\end{array}$ & 2541 & $\begin{array}{l}\text { 1. } \mathrm{HCQ} \\
\text { 2. } \mathrm{HCQ}+\mathrm{AZ} \\
\text { 3. } \mathrm{AZ} \\
\text { 4. SOC }\end{array}$ & $\begin{array}{l}\text { Overall in-hospital mortality } \\
\text { lower in all treatment groups: } \\
\text { HCQ+AZ: } 20.1 \%(95 \% \mathrm{Cl} 17.3 \%- \\
23.0 \%) \\
\text { HCQ: } 13.5 \%(95 \% \mathrm{Cl} 11.6 \%-15.5 \%) \\
\text { AZ: } 22.4 \%(95 \% \mathrm{Cl} 16.0 \%-30.1 \%) \\
\text { SOC: } 26.4 \%(95 \% \mathrm{Cl} 22.2 \%-31.0 \%)\end{array}$ & $\begin{array}{l}\text { - Tx group Pts mean age } 5 \\
\text { y older than in controls } \\
\text { - } \uparrow \text { steroid Tx rates in } \\
\text { HCQ groups } \\
\text { - Missing data due to } \\
\text { reliance only on } \\
\text { electronic health } \\
\text { records }\end{array}$ \\
\hline $\begin{array}{l}\text { Comparative } \\
\text { observational } \\
\text { study }^{10}\end{array}$ & 1438 & $\begin{array}{l}\text { 1. } \mathrm{HCQ} \\
\text { 2. } \mathrm{HCQ}+\mathrm{AZ} \\
\text { 3. } \mathrm{AZ} \\
\text { 4. SOC }\end{array}$ & $\begin{array}{l}\text { No Sig. Dif. in mortality rates for } \\
\mathrm{HCQ}+\mathrm{AZ} \text { (HR } 1.35 ; 95 \% \mathrm{Cl} 0.76- \\
2.40), \mathrm{HCQ} \text { alone (HR } 1.08 ; 95 \% \\
\mathrm{Cl} 0.63-1.85) \text {, or } \mathrm{AZ} \text { alone (HR } \\
0.56 ; 95 \% \mathrm{Cl} 0.26-1.21 \text { ) compared } \\
\text { with control* }\end{array}$ & $\begin{array}{l}\text { - No follow-up of } \\
\text { discharged Pts } \\
\text { - Missing group } \\
\text { characteristics data } \\
\text { - Major difference } \\
\text { between size of SOC vs } \\
\text { Tx groups }\end{array}$ \\
\hline $\begin{array}{l}\text { Comparative } \\
\text { observational } \\
\text { study }^{12}\end{array}$ & 1376 & $\begin{array}{l}\text { 1. } \mathrm{HCQ} \\
\text { 2. } \mathrm{SOC}\end{array}$ & $\begin{array}{l}\text { No significant association } \\
\text { between HCQ and intubation or } \\
\text { death vs SOC (HR 1.04; } 95 \% \mathrm{Cl} \\
0.82-1.32 \text { ) }\end{array}$ & $\begin{array}{l}\text { HCQ Pts more severely ill } \\
\text { than SOC at baseline }\end{array}$ \\
\hline $\begin{array}{l}\text { Comparative } \\
\text { observational } \\
\text { study of } \\
\text { critically ill } \\
\text { ventilated } \\
\text { patients with } \\
\text { ARDS }\end{array}$ & 568 & $\begin{array}{l}\text { 1. } \mathrm{HCQ} \\
\text { 2. SOC }\end{array}$ & $\begin{array}{l}\text { Primary Outcome } \\
\text { - Significant mortality rate } \\
\text { differences between HCQ } \\
(18.8 \%) \text { and SOC }(45.8 \%) \\
\text { groups; } P<0.001 \\
\text { Secondary Outcomes } \\
\text { - Longer HCQ hospitalization } \\
\text { time before death compared to } \\
\text { SOC ( } P<0.05) \\
\text { - IL-6 levels significantly lower } \\
\text { during Tx period for HCQ } \\
\text { oroun. } S O C \text { oroun unchanoed }\end{array}$ & $\begin{array}{l}\text { - Such dramatic } \downarrow \text { in } \\
\text { mortality rate not } \\
\text { described in any other } \\
\text { study } \\
\text { - Different rates of } \\
\text { antibiotics use and } \\
\text { interferon imply } \\
\text { inherent selection bias } \\
\text { for HCQ vs SOC (HCQ } 0 \% \\
\text { interferon vs } 10.8 \% \text { in } \\
\text { SOC }(P=0.01)\end{array}$ \\
\hline
\end{tabular}

Continued on next page 
Table 1. continued

\begin{tabular}{|c|c|c|c|c|}
\hline Study Design & Pts & Study Groups & $\begin{array}{c}\text { Results: Primary/Secondary* } \\
\text { Outcomes }\end{array}$ & Methodological Issues \\
\hline $\begin{array}{l}\text { Comparative } \\
\text { observational } \\
\text { study }^{16}\end{array}$ & 72 & $\begin{array}{l}\text { 1. HCQ-asymptomatic } \\
\text { 2. SOC-asymptomatic }\end{array}$ & $\begin{array}{l}\text { - No Sig. Dif. in recovery rates } \\
\text { (HCQ } 97.5 \% \text { vs SOC } 96.85 \%) \\
\text { - Earlier Tx group recovery ( } 5.4 \\
\text { days) vs SOC ( } 7.6 \text { days) }\end{array}$ & $\begin{array}{l}\text { - HCQ efficiency only } \\
\text { assessed in } \\
\text { asymptomatic Pts } \\
\text { - Inclusion to SOC due to } \\
\text { HCQ contraindications } \\
\text { that probably imply } \\
\text { underlying medical } \\
\text { conditions }\end{array}$ \\
\hline $\begin{array}{l}\text { Comparative } \\
\text { observational } \\
\text { study of only } \\
\text { electronic } \\
\text { health } \\
\text { records }^{13}\end{array}$ & 368 & $\begin{array}{l}\text { 1. } \mathrm{HCQ} \\
\text { 2. } \mathrm{HCQ}+\mathrm{AZ} \\
\text { 3. } \mathrm{SOC}\end{array}$ & 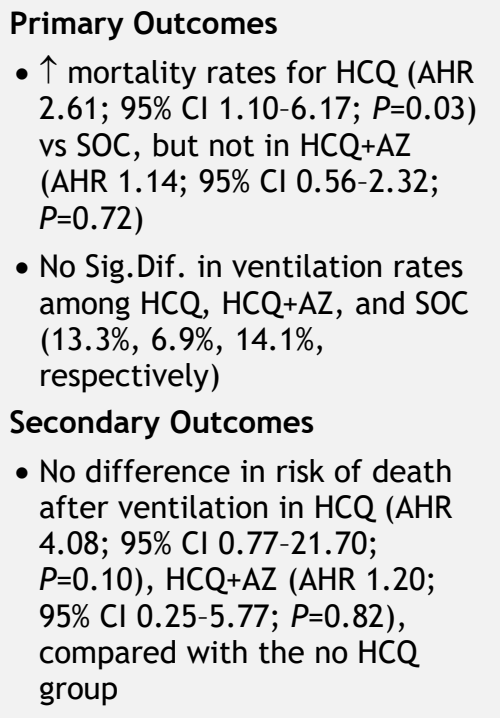 & $\begin{array}{l}\text { - Missing data due to } \\
\text { reliance on electronic } \\
\text { health record codes } \\
\text { - }-95 \% \text { males in all groups }\end{array}$ \\
\hline $\begin{array}{l}\text { Comparative } \\
\text { observational } \\
\text { study of } \\
\text { pneumonia } \\
\text { patients } \\
\text { requiring } \mathrm{O}_{2} \\
\text { without ICU } \\
\text { admission }^{11}\end{array}$ & 181 & $\begin{array}{l}\text { 1. } \mathrm{HCQ} \\
\text { 2. } \mathrm{SOC}\end{array}$ & $\begin{array}{l}\text { Primary Outcome } \\
\text { - No Sig.Dif. in survival rates at } \\
\text { day } 21 \text { for Pts not transferred } \\
\text { to the ICU; } \mathrm{HCQ} 76 \% \text { vs SOC } 75 \% \\
\text { (WHR } 0.9 ; 95 \% \mathrm{Cl} 0.4-2.1 \text { ) } \\
\text { Secondary Outcomes } \\
\text { - No Sig.Dif. in overall survival at } \\
\text { day } 21: \mathrm{HCQ}(89 \%) \text { vs SOC (91\%) } \\
\text { (WHR 1.2; } 95 \% \mathrm{Cl} 0.4-3.3 \text { ) } \\
\text { - No SC for } \mathrm{O}_{2} \text { weaning at day 21: } \\
\mathrm{HCQ} 82 \% \text { vs SOC } 76 \% \text { (WRR 1.1; } \\
95 \% \mathrm{Cl} 0.9-1.3 \text { ) }\end{array}$ & $\begin{array}{l}\text { - HCQ Pts with fewer } \\
\text { comorbidities } \\
\text { - No Tx allocation } \\
\text { protocols }\end{array}$ \\
\hline $\begin{array}{l}\text { Comparative } \\
\text { observational } \\
\text { study }^{17}\end{array}$ & 84 & $\begin{array}{l}\text { 1. } \mathrm{HCQ} \\
\text { 2. } \mathrm{SOC}\end{array}$ & $\begin{array}{l}\text { No Sig. Dif. for: } \\
\text { - Reducing unfavorable outcome } \\
\text { risk (defined as death, ICU } \\
\text { admission, or decision to } \\
\text { withdraw or withhold life- } \\
\text { sustaining treatments) (HR } \\
\text { 0.90; } 95 \% \text { Cl } 0.38-2.1 ; P=0.81 \text { ) } \\
\text { - Overall survival (HR 0.89; } 95 \% \\
\text { Cl } 0.23-3.47 ; P=1 \text { ) }\end{array}$ & Small-scale study \\
\hline
\end{tabular}

Continued on next page 
Table 1. continued

\begin{tabular}{|c|c|c|c|c|}
\hline Study Design & Pts & Study Groups & $\begin{array}{c}\text { Results: Primary/Secondary* } \\
\text { Outcomes }\end{array}$ & Methodological Issues \\
\hline $\begin{array}{l}\text { Comparative } \\
\text { observational } \\
\text { study }^{19}\end{array}$ & 65 & $\begin{array}{l}\text { 1. } \mathrm{HCQ} \\
\text { 2. Lopinavir-ritonavir }\end{array}$ & $\begin{array}{l}\text { Primary Outcome } \\
\text { - Significantly shorter time to } \\
\text { virological cure in lopinavir- } \\
\text { ritonavir group (median } 21 \text { days } \\
\text { vs } 28 \text { days; } P=0.029 \text { ) } \\
\text { Secondary Outcome } \\
\text { - No Sig. Dif. in time to clinical } \\
\text { improvement between groups } \\
\text { (median } 18 \text { days vs } 21 \text { days; } \\
P=0.216 \text { ) }\end{array}$ & $\begin{array}{l}\text { More pneumonia Pts in } \\
\text { lopinavir-ritonavir group }\end{array}$ \\
\hline $\begin{array}{l}\text { Comparative } \\
\text { observational } \\
\text { study }^{20}\end{array}$ & 60 & $\begin{array}{l}\text { 1. Azithromycin, } \\
\text { prednisolone, } \\
\text { naproxen, and } \\
\text { lopinavir/ritonavir } \\
\text { 2. Meropenem, } \\
\text { levofloxacin, } \\
\text { vancomycin, HCQ, and } \\
\text { oseltamivir }\end{array}$ & $\begin{array}{l}\text { Primary Outcome } \\
\text { - } \mathrm{SpO}_{2} \text { saturation, body } \\
\text { temperature, and CRP values } \\
\text { more favorable in Group } 1 \mathrm{Pts} \\
(P=0.013, P=0.012, P<0.001 \text {, } \\
\text { respectively). } \\
\text { Secondary Outcome } \\
\text { - Significantly } \downarrow \text { length of } \\
\text { hospitalization for Group } 1 \text { vs } \\
\text { Group } 2 \text { ( } P=0.001)\end{array}$ & $\begin{array}{l}\text { - Due to multiple drug } \\
\text { regimens, unclear } \\
\text { relative contribution of } \\
\text { each drug to the results } \\
\text { - Adding broad-spectrum } \\
\text { antibiotic to COVID-19 is } \\
\text { probably unnecessary }{ }^{21}\end{array}$ \\
\hline $\begin{array}{l}\text { Uncontrolled } \\
\text { observational } \\
\text { study } 9\end{array}$ & 1061 & 1. $\mathrm{HCQ}+\mathrm{AZ}$ & $\begin{array}{l}\text { - Poor clinical outcome for } 46 \\
\text { Pts }(4.3 \%), 8 \text { died }(0.75 \%), 6 \\
\text { transferred to ICU }(0.56 \%) ; 30 \\
\text { hospital stays >10 days }(2.8 \%) \\
\text { - Prolonged viral carriage in } 47 \\
\text { Pts }(4.4 \%)\end{array}$ & $\begin{array}{l}\text { - Uncontrolled study } \\
\text { - Relatively young Pts: } \\
\text { mean age } 43.6 \text { y }\end{array}$ \\
\hline $\begin{array}{l}\text { Uncontrolled } \\
\text { observational } \\
\text { study }^{8}\end{array}$ & 80 & 1. $H C Q+A Z$ & $\begin{array}{l}\text { - Clinical improvement: } 97.5 \% \\
\text { Pts } \\
\text { - Mean length of hospital stay: } 5 \\
\text { days } \\
\text { - Negative virus cultures in } 97.5 \% \\
\text { Pts at day } 5\end{array}$ & $\begin{array}{l}\text { - Uncontrolled study } \\
\text { - Inadequate length of } \\
\text { post-treatment follow- } \\
\text { up } \\
\text { - Relatively young Pts: } \\
\text { median age } 52.5 \text { y }\end{array}$ \\
\hline
\end{tabular}

* Only primary outcomes are shown, unless the study also provided secondary outcomes.

$\downarrow$, decreased/lower; $\uparrow$, increased/higher; AHR, adjusted hazard ratio; ARDS, acute respiratory distress syndrome; $\mathrm{AZ}$, azithromycin; $\mathrm{Cl}$, confidence interval; CRP, C-reactive protein; $\mathrm{HCQ}$, hydroxychloroquine; HR, hazard ratio; ICU, intensive care unit; $\mathrm{O}_{2}$, oxygen; Pts, patients; Sig.Dif., significant difference(s); SOC, standard of care; $\mathrm{SpO}_{2}$, oxygen saturation; Tx, treatment group; WHR, weighted hazard ratio; WRR, weighted risk ratio.

An independent appraisal study describing a reanalysis of the original Gautret dataset ${ }^{26}$ was recently published by Intson et al. ${ }^{27}$ By excluding all missing datasets, the authors found no differences in viral clearance rates between the treatment and control groups on days 3, 4, 5, or 6. Reproducibility is also a major issue in the Gautret study, as another open-label trial by Molina et al. ${ }^{28}$ used the same dosing regimen and reported that virological cure rates at day 5-6 were only $20 \%$, as opposed to the $70 \%$ reported by Gautret et al.

Another unusual occurrence was the retraction of a large multinational registry analysis published in The Lancet. ${ }^{29}$ This study contained the data of tens of thousands of patients and demonstrated a significant increase of in-hospital mortality rates among the HCQ-treated group compared to patients not 
Table 2. Summary of Randomized Control Trials (RCT) for Evaluating HCQ for COVID-19 Treatment.

\begin{tabular}{|c|c|c|c|c|}
\hline Study Design & Pts & $\begin{array}{l}\text { Study } \\
\text { Groups }\end{array}$ & Results: Primary/Secondary Outcomes & Methodological Issues \\
\hline $\mathrm{RCT}^{24}$ & 150 & $\begin{array}{l}\text { 1. } \mathrm{HCQ} \\
\text { 2. SOC }\end{array}$ & $\begin{array}{l}\text { No Sig. Dif. in virological cure rates at } \\
\text { day } 28\end{array}$ & Open-label study \\
\hline $\mathrm{RCT}^{23}$ & 30 & $\begin{array}{l}\text { 1. } \mathrm{HCQ} \\
\text { 2. SOC }\end{array}$ & $\begin{array}{l}\text { Primary Outcome } \\
\text { - No Sig.Dif. in virological cure rates } \\
\text { (HCQ } 86.7 \% \text { vs SOC } 93.3 \% \text {; } P>0.05 \text { ) } \\
\text { Secondary Outcome } \\
\text { - No Sig.Dif. for time to body } \\
\text { temperature normalization and } \\
\text { improvement in follow-up } \\
\text { examinations }\end{array}$ & $\begin{array}{l}\text { - Published only in Chinese } \\
\text { - Small-scale study }\end{array}$ \\
\hline $\begin{array}{l}\text { Preliminary } \\
\text { data from } \\
\mathrm{RCT}^{22}\end{array}$ & 4674 & $\begin{array}{l}\text { 1. } \mathrm{HCQ} \\
\text { 2. SOC }\end{array}$ & $\begin{array}{l}\text { Primary Outcome } \\
\text { - No Sig.Dif. in survival rates at } 28 \text { days } \\
(25.7 \% \text { vs } 23.5 \% \text { in treatment versus } \\
\text { SOC, HR } 1.11 \text { [ } 95 \% \mathrm{Cl} 0.98-1.26] \text {; } \\
P=0.10) \\
\text { Secondary Outcome } \\
\text { - No impact of HCQ Tx on length of } \\
\text { hospitalization }\end{array}$ & $\begin{array}{l}\text { Preliminary results; not yet } \\
\text { peer-reviewed }\end{array}$ \\
\hline $\mathrm{RCT}^{25}$ & 667 & $\begin{array}{l}\text { 1. } \mathrm{HCQ} \\
\text { 2. } \mathrm{HCQ}+\mathrm{AZ} \\
\text { 3. } \mathrm{SOC}\end{array}$ & $\begin{array}{l}\text { Primary Outcome } \\
\text { - No clinical status benefit at } 15 \text { days } \\
\text { Secondary Outcome } \\
\text { - No mortality benefit } \\
\text { - For HCQ Pts and HCQ+AZ: Increased } \\
\text { incidence of QTC prolongation and } \\
\text { elevated liver enzymes in both } \\
\text { treatment groups when compared to } \\
\text { SOC }\end{array}$ & \\
\hline
\end{tabular}

$A Z$, azithromycin; $\mathrm{Cl}$, confidence interval; $\mathrm{HCQ}$, hydroxychloroquine; HR, hazard ratio; Sig.Dif., significant difference(s); SOC, standard of care.

treated with HCQ. The study was retracted at the request of three of the co-authors, following the refusal of Surgisphere Corporation, which initially provided the patient dataset, to transfer the full dataset, client contracts, and International Organization for Standardization (ISO) audit reports for re-analysis. ${ }^{30}$

\section{Randomized controlled trials}

Two small open-label RCTs comparing HCQ treatment to control patients who did not receive HCQ treatment were conducted in China. Both showed no significant differences in virological cure between the control and treatment groups. ${ }^{23,24}$ Chen et al. found no benefits of HCQ treatment in terms of the median time for body temperature normalization, radiological progression on CT images, and clinical improvement ${ }^{23}$; Tang et al. found no significant difference in the virological cure rate on day 28 with HCQ treatment. ${ }^{24}$

A large RCT conducted in Brazil showed no benefit for HCQ-treated patients and reported a higher incidence of QTc prolongation and elevation of liver enzymes among treated patients. 25

Preliminary results of the large British RECOVERY RCT were recently released to the public before peer review. This study showed no significant differences in the lengths of hospital stay and the death rates at 28 days between the HCQ-treated group and controls. ${ }^{22}$ 
Table 3. Studies with Severe Methodological Issues Evaluating HCQ for COVID-19 Treatment.

\begin{tabular}{|c|c|c|c|c|}
\hline Study Design & Pts & Study Groups & $\begin{array}{c}\text { Results: } \\
\text { Primary/Secondary* } \\
\text { Outcomes }\end{array}$ & Methodological Issues \\
\hline $\begin{array}{l}\text { Comparative } \\
\text { multinational } \\
\text { registry analysis } 29\end{array}$ & 96,032 & $\begin{array}{l}\text { 1. } \mathrm{CQ} \\
\text { 2. } \mathrm{CQ}+\text { macrolide } \\
\text { 3. } \mathrm{HCQ} \\
\text { 4. } \mathrm{HCQ}+\text { macrolide } \\
\text { 5. SOC }\end{array}$ & $\begin{array}{l}\text { Primary Outcome } \\
\text { - Significant } \uparrow \text { of in-hospital } \\
\text { mortality rates for CQ } \\
(16.4 \%) ; \mathrm{CQ}+\text { macrolide } \\
(22.2 \%) ; \mathrm{HCQ}(18.0 \%) ; \\
\text { HCQ+macrolide }(23.8 \%) \text {; } \\
\text { vs SOC }(9.3 \%) \\
\text { Secondary Outcome } \\
\text { - All Tx groups } \\
\text { independently associated } \\
\text { with } \uparrow \text { risk of de novo } \\
\text { ventricular arrhythmia } \\
\text { during hospitalization }\end{array}$ & $\begin{array}{l}\text { - Publication retracted as } \\
\text { per request of three co- } \\
\text { authors } \\
\text { - The corporation that } \\
\text { initially provided the Pt } \\
\text { dataset refused to transfer } \\
\text { the full dataset, client } \\
\text { contracts, and ISO audit } \\
\text { reports for re-analysis }\end{array}$ \\
\hline $\begin{array}{l}\text { Comparative } \\
\text { observational } \\
\text { study }^{26}\end{array}$ & 42 & $\begin{array}{l}\text { 1. } \mathrm{HCQ} \\
\text { 2. } \mathrm{SOC}\end{array}$ & $\begin{array}{l}\text { Sig. Dif. in virological cure } \\
\text { rates between HCQ }(70 \%) \\
\text { and SOC }(12.5 \%) \text { groups } \\
(P=0.001)\end{array}$ & $\begin{array}{l}\text { - Selection bias: SOC group } \\
\text { comprised Pts who refused } \\
\text { HCQ Tx } \\
\text { - Failure to follow-up Pts for } \\
\text { adequate post-treatment } \\
\text { periods } \\
\text { - Age difference between } \\
\text { HCQ and SOC groups: mean } \\
\text { age } 51.2 \text { years vs } 37.3 \\
\text { years, respectively ( } P=0.06) \\
\text { - Unclear exclusion criteria } \\
\text { for HCQ group } \\
\text { - } 5 \text { SOC Pts with no } \\
\text { virological test on day } 6 \\
\text { were included in the final } \\
\text { analysis as COVID-19- } \\
\text { positive cases }\end{array}$ \\
\hline $\begin{array}{l}\text { Re-analysis of } \\
\text { study, }{ }^{26} \text { including } \\
\text { the missing } \\
\text { datasets }^{27}\end{array}$ & 42 & $\begin{array}{l}\text { 1. } \mathrm{HCQ} \\
\text { 2. } \mathrm{SOC}\end{array}$ & $\begin{array}{l}\text { No significant differences in } \\
\text { virological cure rates on } \\
\text { treatment days } 3,4,5 \text {, or } 6 \text { * }\end{array}$ & $\begin{array}{l}\text { None. Study refers to } \\
\text { methodological problems of } \\
\text { the abovementioned study }\end{array}$ \\
\hline $\begin{array}{l}\text { Uncontrolled } \\
\text { observational } \\
\text { study }{ }^{28} \text { aimed at } \\
\text { replicating the } \\
\text { above compara- } \\
\text { tive observational } \\
\text { study }{ }^{26}\end{array}$ & 11 & 1. $\mathrm{HCQ}$ & $\begin{array}{l}20 \% \text { of patients were } \\
\text { virologically cured at day } 5 \text { - } \\
6 \text { post-inclusion* }\end{array}$ & \\
\hline
\end{tabular}

* Only primary outcomes are shown, unless the study also provided secondary outcomes.

$\uparrow$, increased/higher; CQ, chloroquine; ISO, International Organization for Standardization; HCQ, hydroxychloroquine; Pt(s), patient(s); RT-qPCR, reverse transcription polymerase chain reaction; Sig.Dif., significant difference(s); SOC, standard of care; Tx, treatment. 


\section{Studies Evaluating COVID-19 Prevention with HCQ}

Boulware et al. recently published a randomized, double-blind, placebo-controlled trial that assessed hydroxychloroquine use as a post-exposure prophylaxis agent within 4 days of COVID-19 exposure. ${ }^{31}$ The researchers enrolled adults who had household or occupational exposure to someone with confirmed COVID-19, using a strict exposure definition. Participants received either placebo or HCQ, and the primary outcome was the incidence of either laboratory-confirmed COVID-19 or symptoms compatible with COVID-19 within 14 days. Participants were randomized into two groups: HCQ prophylaxis $(n=414)$ and control (placebo; $n=407)$. The numbers of new COVID-19 cases in the treatment and control groups were 49 and 58 , respectively $(P=0.35)$. Side effects were more common with HCQ than with placebo (40.1\% versus $16.8 \%$ ), but no serious adverse reactions were reported.

\section{Studies Evaluating the Safety of HCQ}

Most adverse reactions of treatment with HCQ are mild and include gastrointestinal adverse effects (nausea, vomiting, loss of appetite, diarrhea), skin rash, and photosensitivity. ${ }^{2}$ Prolonged treatment has been associated with irreversible dose-dependent toxic retinopathy. Cardiac toxicity is rare and includes heart failure, conduction disturbance, and QT prolongation. QT prolongation occurs with increasing incidence among older patients and those treated with other medications that prolong the QT interval, e.g. AZ.33

In this review we focused on HCQ effectiveness in treating COVID-19. However, several observational studies assessed the safety profile of HCQ, mainly focusing on cardiovascular adverse events. The observational study by Rosenberg et al. demonstrated a higher likelihood of cardiac arrest in patients receiving $\mathrm{HCQ}+\mathrm{AZ}$ (adjusted OR 2.13; 95\% CI 1.12-4.05), but not HCQ alone (adjusted OR 1.91; 95\% CI 0.96-3.81) or AZ alone (adjusted OR 0.64; 95\% CI 0.27-1.56). ${ }^{10}$

A special attention was given to cardiac arrythmias and QT prolongation following HCQ treatment. In a non-comparative observational study, 24 (2.3\%) of 1,061 patients treated with HCQ reported mild adverse events and no cardiac arrhythmias. 9 However, many other studies reported prolonged QT intervals among HCQ-treated patients.9,10.24,25,33,34 Of note, one patient developed polymorphic ventricular tachycardia requiring emergent cardioversion, and seven patients required premature termination of therapy. ${ }^{35,36}$ Moreover, in a recently published RCT, rates of QTc prolongation were $14.7 \%, 14.6 \%$, and $1.7 \%$ in patients treated with HCQ+AZ, HCQ alone, or none, respectively. 25

\section{DISCUSSION}

This review aimed to assess the possible beneficial role of HCQ in prophylaxis and treatment of COVID-19. To that end, we performed a PubMed search and then narrowed our review down to the 17 most relevant studies. Most studies assessing the therapeutic role of HCQ were observational, and many had an inherent risk of bias. ${ }^{8-20}$ Overall, these observational trials had inconclusive results. The RCTs that have been published so far point to a lack of benefit, although the results are mixed. A large British study, which is expected to be published soon, showed no benefit of HCQ according to early released data. ${ }^{22}$ Use of HCQ as a prophylactic agent was not beneficial in one well-conducted RCT, indicating that its use for COVID-19 should be abandoned..$^{1}$

The safety profile of HCQ is important to assess considering three observations: the drug has no place in therapy as a prophylactic agent 31 ; its therapeutic benefits are questionable $22,23,25$; and its use has been advocated by key political leaders and on social media platforms. 37 In the few studies that assessed the adverse events of HCQ, mild gastrointestinal side effects were relatively common. The rate of QT prolongation was quite variable $(7 \%-76 \%)$ in the few studies reporting it. Whether this risk is translated into an increased risk for cardiac arrhythmias remains to be seen. Even a relatively small risk of such a major event would translate into a large total number of adverse events, since HCQ, at least in the early days of the COVID-19 pandemic, was administered to tens of thousands of patients. ${ }^{33-36}$

Perhaps more important than the assessment of HCQ itself is the critical assessment of events that have led to where we are today. Official guidelines regarding the use of HCQ vary greatly between countries and have changed with time, making the roller-coaster of pros and cons for HCQ use hard to follow. In early March 2020, the Italian Medicines Agency (AIFA) supported the use of chloroquine and HCQ for treatment of COVID-19.38 Shortly afterward, a personal statement supporting the use HCQ as a prophylactic and therapeutic agent was issued by the president of the United States of America 
(USA). The US Food and Drug Administration (FDA) was quick to follow, and issued an emergency use authorization (EUA) to distribute HCQ and chloroquine for the treatment of some COVID-19 inpatients. 39 By April, however, the FDA cautioned against HCQ and chloroquine use due to ensuing risks of arrhythmias ${ }^{40}$; in May, 2020, an article published in The Lancet demonstrated a significant reduction in COVID-19 survival rates following HCQ use. ${ }^{29}$ This article, in turn, was later retracted but only after France, Italy, and Belgium banned the use of HCQ treatment for COVID-19 patients. In June, the preliminary results of the RECOVERY Trial were published demonstrating that HCQ is ineffective in COVID-19 patients compared to control, ${ }^{22}$ and the FDA quickly revoked the EUA for both HCQ and chloroquine. 39 The US Centers for Disease Control and Prevention is currently recommending against the use of chloroquine or HCQ for the treatment of COVID-19, with the exception of patients included in clinical trials. The final results of a large and wellconducted RCT have still not been made fully public. ${ }^{41}$

Several factors have prevented a quicker and more thorough assessment of HCQ over the past few months. The most astonishing fact, when one considers the urgent need for reliable research during a global pandemic, is the fragmentation of scientific efforts between and within countries. Many lowquality observational trials and very few RCTs were conducted. Even now, 7 months after the first cases were reported from Wuhan, more than 300 clinical trials assessing HCQ for COVID-19 are listed in the clinical trial.gov database. Most of these studies will probably never be completed. Instead of creating a few large national or international RCTs with uniform protocols that could later be meta-analyzed, hundreds of different protocols have been created, wasting time and resources.

Large research networks should be created in advance and activated quickly when the need arises. The yet-unpublished British RECOVERY Trial may be the finest example of such an effort during this pandemic. ${ }^{22}$

Selection bias, i.e. including young and relatively healthy patients who are not expected to die from the infection, was also a major contributor to the rendering of the results of several studies as essentially irrelevant for severely ill COVID-19 patients. ${ }^{8,9,14}$ The "corona publication rush" led some scientists to submit studies with major methodological flaws.
Listing a patient death as a "loss to follow-up" may be an extreme example, ${ }^{26}$ but numerous other methodological issues are seemingly abundant. Observational studies are inherently inadequate when the efficacy of a drug is to be assessed, but even in the few RCTs that were conducted, the randomization and assignment procedures were unclear and may have been biased. ${ }^{23,24}$

Some controlled studies compared populations that were different, an inherent limitation of observational studies. For example, a significant difference in the mean age or the proportion of patients receiving steroids (a medication that has been shown to be beneficial to some patients) 37 may have influenced the results.15,26 Drug safety assessments, critical to any trial that involves drug administration for a new indication, were absent from some studies. Shortcomings of the peer review procedures of medical journals were evident. On the one hand, slow peer review meant that initial results, often skewed, inconsistent, or just plain wrong, were available on sites such as medRXiv and the social media platforms many weeks before actual publication. On the other hand, efforts to fast-track what was perceived to be important data led to studies with major methodological flaws being accepted for publication, ${ }^{26}$ and other studies were accepted only to be retracted later. It is crucial for studies that assess data, which could have a major immediate impact on patient management, to be peer-reviewed with a strict protocol that ensures both speed and quality.

The scientific community possesses unprecedented powerful research capabilities owing to cutting-edge technologies, global data sharing, and computational analysis capabilities never previously seen. In theory, these advances should have enabled the scientific community to answer what is, ultimately, a very simple question: is hydroxychloroquine helpful for treating COVID-19? The answer for HCQ as prophylaxis for COVID-19 has been adequately answered, albeit with a no-benefit conclusion. Is hydroxychloroquine beneficial for the treatment of millions of COVID-19 patients? Probably not, but sadly, more than 7 months and 600,000 deaths into the pandemic, that question still has not been definitively answered.

\section{REFERENCES}

1. Worldometer - Real Time World Statistics. Covid-19 Coronavirus Pandemic. Available at: https://www. 
worldometers.info/coronavirus/ (accessed July 23, 2020).

2. Devaux CA, Rolain JM, Colson P, Raoult D. New insights on the antiviral effects of chloroquine against coronavirus: what to expect for COVID-19? Int J Antimicrob Agents 2020;55:105938. $\underline{\text { CrossRef }}$

3. Ng ML, Tan SH, See EE, Ooi EE, Ling AE. Proliferative growth of SARS coronavirus in Vero E6 cells. J Gen Virol 2003;84:3291-303. CrossRef

4. Fantini J, Di Scala C, Chahinian H, Yahi N. Structural and molecular modelling studies reveal a new mechanism of action of chloroquine and hydroxychloroquine against SARS-CoV-2 infection. Int J Antimicrob Agents 2020;55:105960. $\underline{\text { CrossRef }}$

5. Savarino A, Lucia MB, Rastrelli E, et al. Anti-HIV effects of chloroquine: inhibition of viral particle glycosylation and synergism with protease inhibitors. J Acquir Immune Defic Syndr 2004;35:223-32. CrossRef

6. Liu J, Cao R, Xu M, et al. Hydroxychloroquine, a less toxic derivative of chloroquine, is effective in inhibiting SARS-CoV-2 infection in vitro. Cell Discov 2020; 6:16. $\underline{\text { CrossRef }}$

7. Yao X, Ye F, Zhang M, et al. In vitro antiviral activity and projection of optimized dosing design of hydroxychloroquine for the treatment of severe acute respiratory syndrome coronavirus 2 (SARS-CoV-2). Clin Infect Dis 2020; ciaa237. Online ahead of print. CrossRef

8. Gautret P, Lagier JC, Parola P, et al. Clinical and microbiological effect of a combination of hydroxychloroquine and azithromycin in 80 COVID-19 patients with at least a six-day follow up: a pilot observational study. Travel Med Infect Dis 2020;34: 101663. CrossRef

9. Million M, Lagier JC, Gautret P, et al. Early treatment of COVID-19 patients with hydroxychloroquine and azithromycin: a retrospective analysis of 1061 cases in Marseille, France. Travel Med Infect Dis 2020; 35:101738. $\underline{\text { CrossRef }}$

10. Rosenberg ES, Dufort EM, Udo T, et al. Association of treatment with hydroxychloroquine or azithromycin with in-hospital mortality in patients with COVID-19 in New York State. JAMA 2020;323:2493502. $\underline{\text { CrossRef }}$

11. Mahevas M, Tran VT, Roumier M, et al. Clinical efficacy of hydroxychloroquine in patients with covid19 pneumonia who require oxygen: observational comparative study using routine care data. BMJ 2020;369:m1844. $\underline{\text { CrossRef }}$

12. Geleris J, Sun Y, Platt J, et al. Observational study of hydroxychloroquine in hospitalized patients with Covid-19. N Engl J Med 2020;382:2411-18. $\underline{\text { CrossRef }}$
13. Magagnoli J, Narendran S, Pereira F, et al. Outcomes of hydroxychloroquine usage in United States veterans hospitalized with Covid-19. Med 2020; June 5 (In Press). $\underline{\text { CrossRef }}$

14. Yu B, Li C, Chen P, et al. Low dose of hydroxychloroquine reduces fatality of critically ill patients with COVID-19. Sci China Life Sci 2020;1-7. Online ahead of print. CrossRef

15. Arshad S, Kilgore P, Chaudhry ZS, et al. Treatment with hydroxychloroquine, azithromycin, and combination in patients hospitalized with COVID-19. Int J Infect 2020;97:396-403. CrossRef

16. Bhandari S, Singh A, Sharma R, et al. Characteristics, treatment outcomes and role of hydroxychloroquine among 522 COVID-19 hospitalized patients in Jaipur City: an epidemio-clinical study. J Assoc Physicians India 2020;68:13-19.

17. Paccoud O, Tubach F, Baptiste A, et al. Compassionate use of hydroxychloroquine in clinical practice for patients with mild to severe Covid-19 in a French university hospital. Clin Infect Dis 2020;ciaa791. Online ahead of print. CrossRef

18. Lagier JC, Million M, Gautret P, et al. Outcomes of 3,737 COVID-19 patients treated with hydroxychloroquine/azithromycin and other regimens in Marseille, France: a retrospective analysis. Travel Med Infect Dis 2020:101791. CrossRef

19. Kim JW, Kim EJ, Kwon $\mathrm{HH}$, et al. Lopinavirritonavir versus hydroxychloroquine for viral clearance and clinical improvement in patients with mild to moderate coronavirus disease 2019. Korean J Intern Med 2020; June 16. Online ahead of print. $\underline{\text { CrossRef }}$

20. Vahedi E, Ghanei M, Ghazvini A, et al. The clinical value of two combination regimens in the management of patients suffering from Covid-19 pneumonia: a single centered, retrospective, observational study. Daru 2020; June 19;1-10. Online ahead of print. CrossRef

21. Lansbury L, Lim B, Baskaran V, Lim WS. Coinfections in people with COVID-19: a systematic review and meta-analysis. J Infect 2020;81:266-75. CrossRef

22. RECOVERY; Randomised Evaluation of COVID-19 Therapy trial. No clinical benefit from use of hydroxychloroquine in hospitalised patients with COVID-19. Available at: https://www.recoverytrial. net/news/statement-from-the-chief-investigators-ofthe-randomised-evaluation-of-covid-19-therapyrecovery-trial-on-hydroxychloroquine-5-june-2020no-clinical-benefit-from-use-of-hydroxychloroquinein-hospitalised-patients-with-covid-19 (accessed July 23, 2020). 
23. Chen J, Liu D, Liu L, et al. [A pilot study of hydroxychloroquine in treatment of patients with moderate COVID-19]. Zhejiang Da Xue Xue Bao Yi Xue Ban 2020;49:215-19. Chinese.

24. Tang W, Cao Z, Han M, et al. Hydroxychloroquine in patients with mainly mild to moderate coronavirus disease 2019: open label, randomised controlled trial. BMJ 2020;369:m1849. CrossRef

25. Cavalcanti AB, Zampieri FG, Rosa RG, et al. Hydroxychloroquine with or without azithromycin in mild-to-moderate Covid-19. N Engl J Med 2020; Jul 23. Online ahead of print. $\underline{\text { CrossRef }}$

26. Gautret P, Lagier JC, Parola P, et al. Hydroxychloroquine and azithromycin as a treatment of COVID-19: results of an open-label non-randomized clinical trial. Int J Antimicrob Agents 2020:105949. Online ahead of print. $\underline{\text { CrossRef }}$

27. Intson K, Kumar S, Botta A, Neckles R, Leung C, Jawaid A. An independent appraisal and re-analysis of hydroxychloroquine treatment trial for COVID-19. Swiss Med Wkly 2020;150:w20262. CrossRef

28. Molina JM, Delaugerre C, Le Goff J, et al. No evidence of rapid antiviral clearance or clinical benefit with the combination of hydroxychloroquine and azithromycin in patients with severe COVID-19 infection. Med Mal Infect 2020;50:384. $\underline{\text { CrossRef }}$

29. Mehra MR, Desai SS, Ruschitzka F, Patel AN. RETRACTED: Hydroxychloroquine or chloroquine with or without a macrolide for treatment of COVID19: a multinational registry analysis. Lancet 2020; So140-6736(20)31180-6. CrossRef

30. Mehra MR, Ruschitzka F, Patel AN. RetractionHydroxychloroquine or chloroquine with or without a macrolide for treatment of COVID-19: a multinational registry analysis. Lancet 2020;395:1820. CrossRef

31. Boulware DR, Pullen MF, Bangdiwala AS, et al. A randomized trial of hydroxychloroquine as postexposure prophylaxis for Covid-19. N Engl J Med 2020;NEJMoa2016638. Online ahead of print. CrossRef

32. Plantone D, Koudriavtseva T. Current and future use of chloroquine and hydroxychloroquine in infectious, immune, neoplastic, and neurological diseases: a mini-review. Clin Drug Investig 2018;38:653-71. $\underline{\text { CrossRef }}$

33. Mercuro NJ, Yen CF, Shim DJ, et al. Risk of QT interval prolongation associated with use of hydroxychloroquine with or without concomitant azithromycin among hospitalized patients testing positive for coronavirus disease 2019 (COVID-19). JAMA Cardiol 2020;e201834. $\underline{\text { CrossRef }}$
34. Painvin B, Guillot P, Verdier MC, Gacouin A, Maamar A. Hydroxychloroquine pharmacokinetic in COVID19 critically ill patients: an observational cohort study. Intensive Care Med 2020; June 8:1-2. Online ahead of print. $\underline{\text { CrossRef }}$

35. Chorin E, Wadhwani L, Magnani S, et al. QT interval prolongation and torsade de pointes in patients with COVID-19 treated with hydroxychloroquine/azithromycin. Heart Rhythm 2020;S1547-5271(20)30435-5. CrossRef

36. Chorin E, Dai M, Shulman E, et al. The QT interval in patients with COVID-19 treated with hydroxychloroquine and azithromycin. Nat Med 2020;26:808-9. CrossRef

37. RECOVERY; Randomised Evaluation of COVID-19 Therapy. University of Oxford. Low-cost dexamethasone reduces death by up to one third in hospitalised patients with severe respiratory complications of COVID-19. Available at: https://www.recoverytrial. net/files/recovery dexamethasone statement 1606 20 final.pdf (accessed June 16, 2020).

38. AIFA, Agenzia Italiana del Farmaco Azioni intraprese per favorire la ricerca e l'accesso ai nuovi farmaci per il trattamento del COVID-19. Available at: https:// www.aifa.gov.it/-/azioni-intraprese-per-favorire-laricerca-e-l-accesso-ai-nuovi-farmaci-per-iltrattamento-del-covid-19 (accessed March 17, 2020) (Italian).

39. U.S. Food and Drug Administration (FDA). Coronavirus (COVID-19) update: FDA revokes emergency use authorization for chloroquine and hydroxychloroquine. Available at: https://www.fda.gov/newsevents/press-announcements/coronavirus-covid-19update-fda-revokes-emergency-use-authorizationchloroquine-and (accessed June 15, 2020).

40. U.S. Food and Drug Administration (FDA). FDA cautions against use of hydroxychloroquine or chloroquine for COVID-19 outside of the hospital setting or a clinical trial due to risk of heart rhythm problems. Available at: https://www.fda.gov/drugs/drugsafety-and-availability/fda-cautions-against-usehydroxychloroquine-or-chloroquine-covid-19outside-hospital-setting-or (accessed April 24, 2020).

41. National Institutes of Health (NIH). COVID-19 Treatment Guidelines Panel. Coronavirus Disease 2019 (COVID-19) treatment guidelines. Available at: https://www.covid19treatmentguidelines.nih.gov/ (accessed July 23, 2020).

42. BBC News. Coronavirus: Trump says he is taking unproven drug hydroxychloroquine. 2020; May 19. Available at: https://www.bbc.com/news/world-uscanada-52717161 (accessed July 28, 2020). 